\title{
White Matter Water Diffusion Changes in Primary Sjögren Syndrome
}

\author{
L.C. Tzarouchi, A.K. Zikou, N. Tsifetaki, L.G. Astrakas, S. Konitsiotis, P. Voulgari, A. Drosos, and M.I. Argyropoulou
}

\begin{abstract}
BACKGROUND AND PURPOSE: Histopathologic studies have demonstrated WM damage in primary Sjögren syndrome. The purpose of this study was to evaluate WM microstructural changes by use of DTI-derived parameters in patients with primary Sjögren syndrome.
\end{abstract}

MATERIALS AND METHODS: DTI was performed in 19 patients with primary Sjögren syndrome (age, $64.73 \pm 9.1$ years; disease duration, $11.5 \pm 7.56$ years) and 16 age-matched control subjects. Exclusion criteria were a history of major metabolic, neurologic, or psychiatric disorder and high risk for cardiovascular disease. Data were analyzed by use of tract-based spatial statistics, for which the WM skeleton was created, and a permutation-based inference with 5000 permutations was used with a threshold of $P<.01$, corrected for multiple comparisons to enable identification of abnormalities in fractional anisotropy, mean diffusivity, radial diffusivity, and axial diffusivity.

RESULTS: Tract-based spatial statistics showed decreased fractional anisotropy in multiple areas in patients with primary Sjögren syndrome compared with control subjects, located mainly in the corticospinal tract, superior longitudinal fasciculus, anterior thalamic radiation, inferior fronto-occipital fasciculus, uncinate fasciculus, and inferior longitudinal fasciculus. Increased mean diffusivity and radial diffusivity and decreased axial diffusivity were observed in most of the fiber tracts of the brain in patients with primary Sjögren syndrome, compared with control subjects.

CONCLUSIONS: Patients with primary Sjögren syndrome show loss of WM microstructural integrity, probably related to both Wallerian degeneration and demyelination.

ABBREVIATIONS: $A D=$ axial diffusivity; $F A=$ fractional anisotropy; $M D=$ mean diffusivity; $\mathrm{pSS}$ = primary Sjögren syndrome; RD = radial diffusivity; TBSS = tract-based spatial statistics

S ögren syndrome is a chronic systemic autoimmune disease that can be classified as primary Sjögren syndrome (pSS) when presenting in isolation or secondary when related to another connective tissue disease. ${ }^{1}$ The prevalence of pSS reported in different studies ranges from $0.1-4.8 \% .^{2}$ pSS is characterized by mononuclear infiltration and destruction of the exocrine glands, mainly the lachrymal and salivary glands, but extraglandular manifestations are also reported (eg, arthralgia, pulmonary involvement, renal tubular acidosis, etc). ${ }^{1,3}$ Involvement of both the peripheral and the CNS has also been reported in pSS. ${ }^{4-6} \mathrm{Al}-$ though involvement of the peripheral nervous system is a welldocumented feature of the disease, the prevalence, the type, and the underlying mechanism of CNS involvement remain un-

Received June 13, 2013; accepted after revision August 10.

From the Departments of Radiology (L.C.T., A.K.Z., M.I.A.), Rheumatology (N.T., P.V., A.D.), Medical Physics (L.G.A.), and Neurology (S.K.), Medical School, University of loannina, Greece.

Please address correspondence to Maria I. Argyropoulou, MD, Medical School University of Ioannina, 45110 loannina, Greece; e-mail: margyrop@cc.uoi.gr

http://dx.doi.org/10.3174/ajnr.A3756 clear. ${ }^{4,5,7}$ The estimated frequency of CNS involvement ranges from $10-60 \%$ in different reports, depending on the parameters studied (eg, patient selection, diagnostic criteria, etc). ${ }^{4-6}$ Patients with pSS can present with a wide range of focal or diffuse neurologic or psychiatric manifestations, including motor/sensory deficits, transverse myelitis, and cognitive impairment. ${ }^{4-6}$ The current data from MRI studies support an increased frequency of high signal intensity lesions in the periventricular and/or subcortical WM on FLAIR and T2-weighted imaging, observed mainly in patients with pSS and evidence of CNS disease. ${ }^{8-11}$ The volumetric analysis of GM and WM by use of the voxel-based morphometry method demonstrated diffuse atrophy in patients with pSS. ${ }^{8}$ SPECT and PET studies have demonstrated reduced CBF and lowered glucose metabolism in patients with pSS. ${ }^{12,13}$

DTI is a technique that allows assessment of the preferential direction of the Brownian motion of protons, which in the brain reflects the microscopic architecture of the WM. ${ }^{14,15}$ Four quantitative diffusion parameters can be derived from DTI data: 1) fractional anisotropy (FA), reflecting the directionality of water diffusion and coherence of WM fiber tracts; 2) mean diffusivity 
Table 1: Demographic and clinical characteristics of 19 patients with pSS

\begin{tabular}{lc}
\hline & Patients With pSS \\
\hline Age, mean \pm SD & $64.73 \pm 9.1$ years \\
Sex, female:male & $19: 0$ \\
Disease duration, mean \pm SD & $11.5 \pm 7.56$ years \\
Dry mouth & $94.7 \%$ \\
Dry eyes & $94.7 \%$ \\
Positive salivary gland biopsy & $100 \%$ \\
Arthritis/arthralgia & $63.2 \%$ \\
Raynaud phenomenon & $47.4 \%$ \\
\hline
\end{tabular}

(MD), quantifying the overall magnitude of water diffusion; 3 ) axial diffusivity (AD), measuring the magnitude of diffusivity along the principal diffusion direction; and 4) radial diffusivity (RD), reflecting the magnitude of diffusivity perpendicular to the principal diffusion direction. ${ }^{14,15}$ These metrics have been correlated with the microstructural organization of WM and are used to infer structural characteristics of the local tissue. ${ }^{14,15}$ The DTIderived parameters have been used to investigate WM microstructure in various disorders by use of an ROI approach, in which structures of interest are manually defined on MR images, but information about changes in brain diffusivity in pSS is scarce. ${ }^{16}$ Using the ROI method, a decrease in FA and an increase in MD values have been reported in patients with pSS. ${ }^{16}$ Similar analytic methods that are based on manually selected ROIs have limitations, mainly because they do not examine the whole brain and they are laborious and time-consuming and therefore prone to human error. ${ }^{17}$ To eliminate the limitations of the ROI-based methods, the so-called voxelwise analysis methods have been developed, which examine the whole brain automatically at a voxel level. ${ }^{17}$ The technique of tract-based spatial statistics (TBSS) allows voxelwise statistical analysis of DTI-derived data. ${ }^{18}$ TBSS has been widely used for DTI analysis because of its advanced registration capabilities and its robust nonparametric assessment of local differences in WM integrity between groups. ${ }^{18}$

The purpose of the present study was to assess the presence and location of WM damage and to elucidate the basis of WM microstructural changes in patients with pSS by analyzing DTIderived parameters with the automated TBSS method.

\section{MATERIALS AND METHODS Study Patients}

The study population consisted of 19 consecutively registered, unselected patients with pSS being followed up in the outpatient rheumatology clinic of our hospital, ages $47-78$ years (mean \pm SD; $64.73 \pm 9.1$ years), with a disease duration of 5-28 years (mean $\pm \mathrm{SD} ; 11.5 \pm 7.56$ years). The diagnosis of $\mathrm{pSS}$ was established according to the American-European Consensus Criteria. Association with other connective tissue diseases was ruled out, and only patients with pSS were included. The demographic and clinical characteristics of the patients are shown in Table 1. The control group consisted of 16 age-matched healthy volunteers, ages $45-76$ years (mean \pm SD; $62.57 \pm 8.3$ years). The protocol and the procedure were explained in detail to all patients and control subjects who had the same educational background. The study was performed with the approval of the institutional review board, and all the participants signed a written informed consent agreement.
Exclusion criteria were a history or clinical signs of cardiovascular disease, peripheral arterial disease, hepatic dysfunction (serum transaminase levels $>1.5$ times the upper limit of normal), renal insufficiency (serum creatinine concentration $>1.6 \mathrm{mg}$ / $\mathrm{dL}$ ), proteinuria $(>0.5 \mathrm{~g} / \mathrm{d})$, diabetes mellitus (fasting plasma glucose concentration $\geq 126 \mathrm{mg} / \mathrm{dL}$ or use of antidiabetic medication), hypertension (arterial blood pressure $>140 / 90 \mathrm{~mm} \mathrm{Hg}$ or use of antihypertensive medication), serum level of thyroid-stimulating hormone $>5 \mathrm{mU} / \mathrm{mL}$, and treatment with corticosteroids during the previous 6 months. None of the study patients or control subjects had findings suggestive of CNS or psychiatric disorder. A total of 30 patients with pSS were initially evaluated. However, 11 were excluded. More specifically, 3 had hypertension for many years, 2 had atrial fibrillation, 4 had diabetes mellitus and dyslipidemia, and 2 had claustrophobia. Thus, 19 patients were finally included. Routine neurologic examination was performed on all subjects and did not reveal deficits indicative of central or peripheral nervous system involvement. Similarly, patients and caregivers did not report any symptoms indicative of cognitive decline, depression, or fatigue.

\section{Data Acquisition}

DTI was performed by use of a 1.5T scanner (Gyroscan ACS NT; Philips Healthcare, Best, The Netherlands). We used a single-shot EPI sequence. Parameters for DTI acquisition were as follows: FOV $=230 \times 230 \mathrm{~mm}, 112 \times 128$ matrix, section thickness of 3 $\mathrm{mm}, \mathrm{TE}=131 \mathrm{~ms}, \mathrm{TR}=9825 \mathrm{~ms}$, number of sections $=42$, section gap $=0 \mathrm{~mm}$. We used 16 noncollinear gradient directions, with maximum $b=700$ seconds $/ \mathrm{mm}^{2}$ and scanning time 4 minutes, 34 seconds. The imaging protocol also included 1) a FLAIR sequence $(\mathrm{TR}=6300 \mathrm{~ms}$, $\mathrm{TE}=120 \mathrm{~ms}, \mathrm{FOV}=250 \mathrm{~mm}$, matrix $=256 \times 256$, section thickness of $6 \mathrm{~mm}$, intersection gap $=$ 0.6 , scanning time $=2$ minutes, 50 seconds), 2) a T1-weighted, high-resolution $(1 \times 1 \times 1 \mathrm{~mm})$, 3D spoiled gradient-echo sequence $(\mathrm{TR}=25 \mathrm{~ms}, \mathrm{TE}=4.6 \mathrm{~ms}$, acquisition matrix $=256 \times$ $228, \mathrm{FOV}=220 \mathrm{~mm}$, scanning time $=5$ minutes, 43 seconds).

The presence of areas of high signal intensity in the WM was assessed for each subject on FLAIR images by 2 neuroradiologists who were blinded to the patient/control status.

\section{Data Preprocessing}

Images were processed by use of the FSL (FMRIB Software Library; http://www.fmrib.ox.ac.uk/fsl) software package. ${ }^{19}$ For each subject, all images including diffusion-weighted and B0 images were corrected for eddy current-induced distortion and subject motion effect by use of the FSL Diffusion Toolbox. Brain mask was created from the first B0 image by use of the FSL Brain Extraction Tool, and Diffusion Toolbox was used to fit the tensor model and to compute the $\mathrm{FA}, \mathrm{MD}, \mathrm{AD}$, and $\mathrm{RD}$ maps.

\section{Tract-Based Spatial Statistics Analysis}

Voxelwise analysis was performed by use of TBSS. ${ }^{18}$ First, every FA image was aligned to every other one; by use of all these comparisons, the software then identified the "most representative" one and used it as the target image. This target image was then affine-aligned into Montreal Neurological Institute 152 standard space. The FA data of all subjects were aligned to this target image 
by use of the nonlinear registration tool FNIRT, which uses a b-spline representation of the registration warp field. Next, the mean FA image was created and thinned to create a mean FA skeleton, which represents the centers of all tracts common to the group. A threshold of FA $>0.2$ was applied to the skeleton to include only major fiber bundles. The aligned FA data of each subject were then projected onto this skeleton. By applying the original nonlinear registration of each subject's FA to standard space, the $\mathrm{MD}, \mathrm{RD}$, and $\mathrm{AD}$ maps were also projected onto the mean FA skeleton. The projected maps were separated into 2 groups (control subjects and patients with pSS) and were used to calculate voxelwise cross-subject diffusion statistics. The localization of all the anatomic information described was based on the Johns Hopkins University WM tractography atlas and the International Consortium for Brain Mapping DTI-81 WM labels (part of the FSL package).

\section{Statistical Analysis}

To determine $\mathrm{FA}, \mathrm{MD}, \mathrm{RD}$, and $\mathrm{AD}$ differences between groups, the voxelwise analysis was performed by a permutation-based inference method as implemented in the Randomize FSL tool. ${ }^{20} \mathrm{We}$ used a $t$ test, 5000 permutations, and threshold-free cluster enhancement with a threshold of $P<.01$, corrected for multiple comparisons by use of family-wise error correction to assess differences in the $\mathrm{FA}, \mathrm{MD}, \mathrm{RD}$, and $\mathrm{AD}$ between the patients with pSS and the healthy control subjects. ${ }^{21}$ In addition, for the patients with pSS, voxelwise associations between each diffusion metric (FA, MD, $\mathrm{AD}$, and $\mathrm{RD}$ ) and disease duration were performed by use of a nonparametric, linear regression model. The significance threshold for correlations was set at $P<.05$, corrected for multiple comparisons by use of family-wise error correction (threshold-free cluster enhancement option in the Randomize permutation-testing tool).

\section{RESULTS}

There was no significant difference in age between patients and control subjects. Areas of high signal intensity in WM were observed in 13 of the 19 patients $(68.4 \%)$ and in 6 of the 16 control subjects $(37.5 \%)$.

\section{Tract-Based Spatial Statistics}

In the voxelwise-based group comparison, multiple WM areas with significant FA decrease $(P<.01$, family-wise error corrections for multiple comparison) were found bilaterally in patients with pSS compared with control subjects, as shown in Fig $1 A$, in the corticospinal tract, the superior longitudinal fasciculus, the anterior thalamic radiation, the inferior fronto-occipital fasciculus, the uncinate fasciculus, and the inferior longitudinal fasciculus (Table 2).

Voxelwise-based group comparison of MD, AD, and RD between patients with pSS and control subjects showed increased $\mathrm{MD}$ and $\mathrm{RD}$ and decreased $\mathrm{AD}$ in the patients in a widespread, diffuse pattern involving most of the major WM tracts throughout the brain (Fig $1 B, 1 C$, and $1 D$, respectively).

Finally, regression analysis did not show any significant association between the DTI metrics and disease duration.

\section{DISCUSSION}

The main findings in the present study were decreased FA values in patients with pSS compared with control subjects in multiple major WM tracts, including the corticospinal tract, the superior longitudinal fasciculus, the anterior thalamic radiation, the inferior fronto-occipital fasciculus, the uncinate fasciculus, and the inferior longitudinal fasciculus. These changes in FA are related to widespread decreased $\mathrm{AD}$ values and increased MD and RD values in patients with pSS in comparison with control subjects.

The decreased FA and the increased MD values found in the present study are in accordance with the findings of the single previous study evaluating DTI in a similar number of patients with pSS ( $n=19)$, which used the ROI method to evaluate 2 areas in the frontal lobes. ${ }^{16}$ The present study adds to these previous findings by use of the TBSS analysis, which permits voxelwise statistical analysis of all DTI data and revealed more extensive changes in patients with pSS. ${ }^{18}$

FA is a measure of the degree to which water diffusion is constrained in the brain, and its primary determinant is the packing attenuation of axons within a voxel. ${ }^{15,22}$ Axonal packing attenuation encompasses a variety of microstructural level variables (eg, degree of myelination, axonal diameters, and extracellular space). ${ }^{15,22}$ The decreased FA values demonstrated in patients with pSS thus indicate loss of WM fiber integrity. MD quantifies the amount of diffusion within a brain voxel, but it lacks directional information, and increased MD values point to an increase overall in directionally nonspecific water diffusivity and suggest tissue breakdown with an increase in brain-water content. ${ }^{15}$

The MD and FA indices allow for quantitative evaluation of the random translational motion of water molecules and have been shown to reflect a variety of pathologic states in the brain, ${ }^{23,24}$ but, despite being sensitive, they are lacking in specificity. The changes in diffusion-tensor eigenvalues $\lambda_{1}, \lambda_{2}$, and $\lambda_{3}$, which are the source indexes for calculating the MD and FA, may provide further information about the underlying neuropathologic mechanisms. ${ }^{25,26}$ The $\lambda_{1}$ or AD measures the diffusion coefficient along the direction of maximum diffusivity and reflects changes in restrictive barriers along the direction of a tract. ${ }^{25,26}$ The $\left(\lambda_{2}+\lambda_{3}\right) / 2$ or RD measures the diffusion coefficient perpendicular to the direction of maximum diffusivity and reflects mainly changes in the axonal membrane and the myelin sheath. ${ }^{25,26}$ It should be noted that $\mathrm{AD}$ and $\mathrm{RD}$, unlike FA, are not rotationally invariant metrics of the diffusion tensor, and therefore their values depend on the orientation of the diffusion tensor ellipsoid at each voxel. Consequently, their usage in multisubject studies requires precise registration that ensures alignment of the different diffusion tensor ellipsoids in every voxel; otherwise, interpretation of changes of the "axial" and "radial" diffusivities on the basis of the underlying tissue structure becomes problematic. ${ }^{27}$ Experimental studies have demonstrated that axonal damage leads to a marked decrease in $\mathrm{AD}$ and modest, often insignificant, decreases in $\mathrm{RD}$, whereas demyelination increases $\mathrm{RD}$ without changing $\mathrm{AD}$ when these phenomena take place in isolation. ${ }^{28,29}$ In the present study, the decreased AD and increased RD may be suggestive of decreased organization of tracts or axonal damage, which prevents diffusion along the long (axial) axis, or alternatively, decreased myelination that allows for more room 


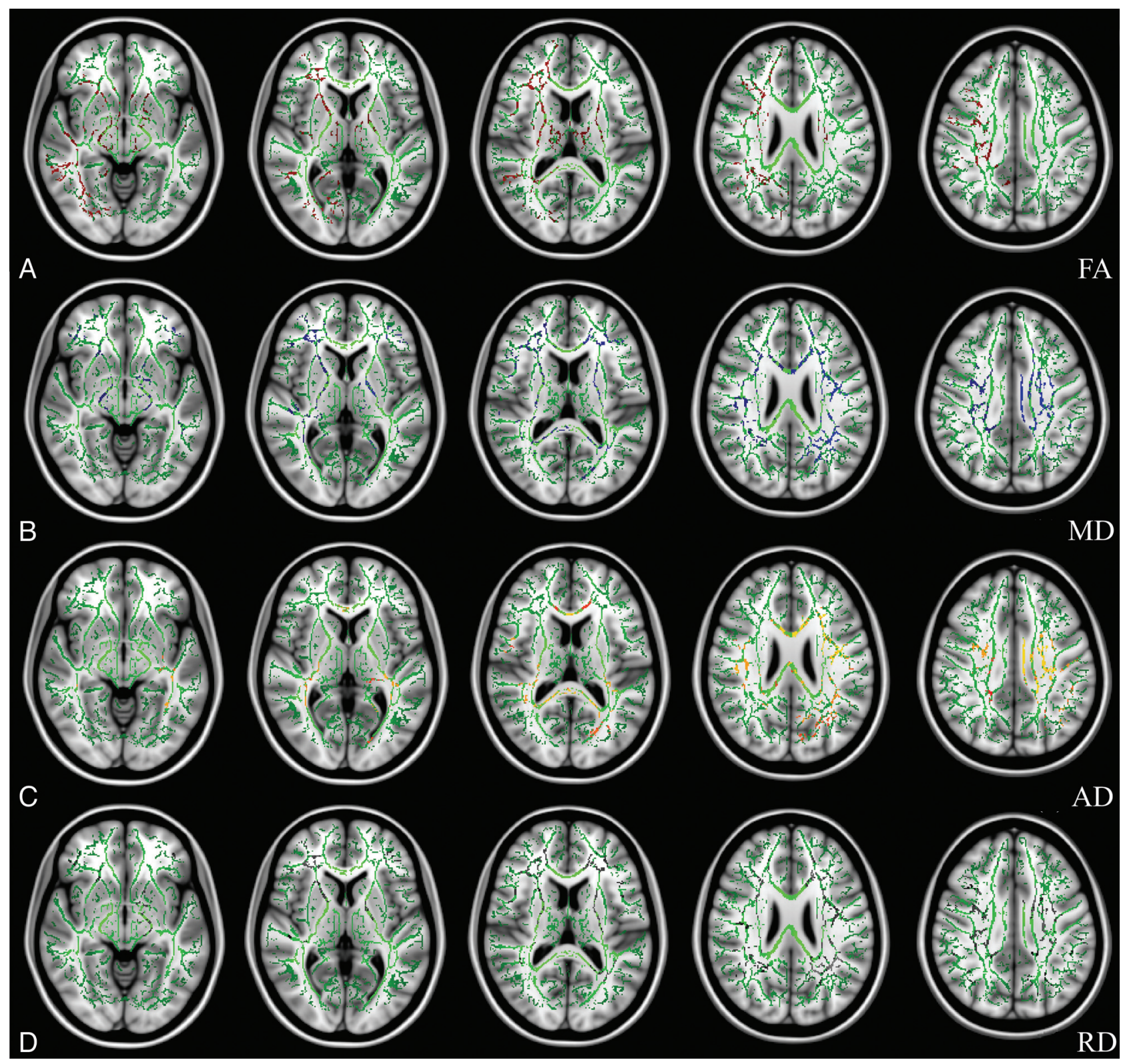

FIG 1. Tract-based spatial statistics results demonstrate voxelwise comparisons between 19 patients with pSS and 16 control subjects. Statistical maps (thresholded at threshold-free cluster enhancement, $P<.01$ ) are overlaid onto the mean FA skeleton and the Montreal Neurological Institute 152 template. Decreased fractional anisotropy in patients with pSS is shown in red (A), increased mean diffusivity is shown in blue (B), decreased axial diffusivity is shown in orange-yellow (C), and increased radial diffusivity is shown in gray (D). A, Corticospinal tract, superior longitudinal fasciculus, anterior thalamic radiation, inferior fronto-occipital fasciculus, uncinate fasciculus, and inferior longitudinal fasciculus; $B$, superior longitudinal fasciculus, thalamic radiation, inferior fronto-occipital fasciculus, uncinate fasciculus and inferior longitudinal fasciculus, corticospinal tract, cingulum, genu, and splenium of the corpus callosum; $C$, superior longitudinal fasciculus, thalamic radiation, inferior frontooccipital fasciculus, uncinate fasciculus and inferior longitudinal fasciculus, corticospinal tract, cingulum, and genu and splenium of the corpus callosum; $D$, superior longitudinal fasciculus, thalamic radiation, inferior fronto-occipital fasciculus, uncinate fasciculus, inferior longitudinal fasciculus, corticospinal tract, and cingulum.

between axons for water molecules to move perpendicular to the tract (ie, radially).

Several pathogenetic mechanisms may account for the findings in the present study: 1) WM involvement could reflect axonal damage through anterograde or Wallerian degeneration. Cerebral small-vessel vasculitis has been reported in patients with pSS, leading to hypoperfusion of the cortex and atrophy. ${ }^{12,13,30,31}$ With the use of voxel-based morphometry, diffuse GM atrophy has been demonstrated in patients with pSS. ${ }^{8}$ This cortical atrophy may induce Wallerian degeneration of WM tracts. ${ }^{8}$ Wallerian degeneration is characterized by a stereotypical course, starting with disintegration of axonal structures within days after injury, followed by fragmentation-degradation of myelin caused by infiltration of macrophages and finally, fibrosis, and atrophy of the affected fiber tracts. ${ }^{32,33}$ Loss of axonal structure may result in less restricted diffusion perpendicular to the main direction of fibers and consequently give rise to elevated RD. ${ }^{22,34}$ The membrane disintegration and cellular debris create new diffusion barriers that lead to a decrease in diffusivity parallel to the main fiber direction and therefore reduced $\mathrm{AD}^{22,34}$;2) WM damage could be 


\begin{tabular}{|c|c|c|c|c|}
\hline \multirow[b]{2}{*}{ Anatomic Region } & \multicolumn{3}{|c|}{ MNI Coordinates, mm } & \multirow[b]{2}{*}{ Cluster Size } \\
\hline & $x$ & $y$ & $z$ & \\
\hline $\begin{array}{l}\text { Right superior corona radiata, superior longitudinal fasciculus, } \\
\text { anterior and posterior limb of internal capsule, inferior } \\
\text { fronto-occipital fasciculus, uncinate fasciculus }\end{array}$ & 27 & 8 & 26 & 4286 \\
\hline Left anterior thalamic radiation & -8 & -18 & 0 & 284 \\
\hline $\begin{array}{l}\text { Left inferior longitudinal fasciculus, uncinate fasciculus, inferior } \\
\text { fronto-occipital fasciculus }\end{array}$ & -46 & 7 & -20 & 202 \\
\hline Right anterior thalamic radiation, inferior longitudinal fasciculus & 10 & -17 & 5 & 103 \\
\hline Left anterior limb of internal capsule, anterior thalamic radiation & -23 & 7 & 14 & 92 \\
\hline $\begin{array}{l}\text { Left superior corona radiata, superior longitudinal fasciculus, } \\
\text { corticospinal tract }\end{array}$ & -28 & -15 & 21 & 73 \\
\hline Left anterior thalamic radiation & -16 & 15 & -6 & 67 \\
\hline Left inferior longitudinal fasciculus, uncinate fasciculus & -44 & 4 & -36 & 65 \\
\hline Left anterior limb of internal capsule, anterior thalamic radiation & -20 & 0 & 11 & 46 \\
\hline Left inferior longitudinal fasciculus & -16 & 3 & -5 & 38 \\
\hline
\end{tabular}

Note:- $P<.01$, family-wise error-corrected. MNI indicates Montreal Neurological Institute.

caused by a direct insult from antibodies against myelin. Recently, an autoantibody targeting the water channel protein aquaporin-4 (anti-aquaporin-4) has been discovered in neuromyelitis optica, leading to death of oligodendrocytes, demyelination, and axonal loss. ${ }^{35}$ It is well known that there is a strong association between pSS and neuromyelitis optica, and there is evidence of an increased prevalence of anti-aquaporin- 4 antibodies in patients with pSS. ${ }^{36-38}$ Loss of myelin reduces the barriers that restrict diffusion perpendicular to the WM fibers, leading to increased $\mathrm{RD} .{ }^{28}$ Axonal damage is an integral part of demyelination, and the concurrent axonal transactions and disruptions associated with axonal damage lead to the addition of diffusion barriers parallel to the axon and thus to reduced AD. ${ }^{39}$ Finally, the underlying process accounting for the WM involvement might be a combination of Wallerian degeneration and demyelination, which is supported by a limited number of autopsy studies that have demonstrated both axonal degeneration and demyelination in the CNS of patients with pSS. ${ }^{40,41}$ Similarly, decreased FA and AD and increased $\mathrm{RD}$ have been demonstrated in patients with neuromyelitis optica. ${ }^{42,43}$ These changes involve multiple, major WM tracts in the normal-appearing WM, accumulate with increasing disease duration, and are probably related to both demyelination and Wallerian degeneration. ${ }^{42,43}$

A global decrease in WM integrity throughout the brain was observed in multiple major fiber tracts that control a wide range of brain functions. The CNS manifestations in pSS are heterogeneous, manifested as focal or diffuse involvement. ${ }^{5,6}$ The wide spectrum of CNS manifestations in pSS includes movement disorders, motor and sensory loss, seizures, cognitive impairment, dementia, psychiatric abnormalities, encephalopathy, optic neuropathies, and others. ${ }^{5,6}$ This wide range of CNS manifestations in patients with pSS is in accordance with the diffuse WM involvement throughout the brain detected by DTI in the present study.

The present study has some certain limitations that must be noted. First, the sample size was relatively small, which might reduce the power of the statistical significance and the generalization of the findings. Second, the cross-sectional design of the study and the reliance on a single imaging technique do not allow for assessment of the relative time that WM integrity is affected. Third, our DTI acquisition sequence had a limited number of directions that might have limited the precision of the values of the DTI parameters. Furthermore, the TBSS analysis that was used in the present study (as in any other analyses) is not without flaws or limitations. One is the lack of accuracy caused by the low-resolution DTI (partial volume effect). However, this is more an acquisition limitation rather than a postprocessing flaw, and TBSS remains one of the most reliable methods for multisubject DTI analysis available today. Finally, the lack of official cognitive or neuropsychiatric testing and reliable correlations with our imaging findings weaken their clinical interpretation/value. Notwithstanding the problematic issues raised, this study adds to the limited existing literature on CNS involvement in patients with pSS and should be considered preliminary. Therefore, future studies in larger populations, as well as prospective, longitudinal studies with the use of additional advanced MR imaging techniques with histopathologic and clinical correlations, will be useful for further probing the nature and the relationship of WM abnormalities to clinical symptoms in pSS.

\section{CONCLUSIONS}

Loss of WM microstructural integrity is demonstrated in patients with pSS as reduced FA and AD values and increased MD and RD values. Involvement of the WM might be caused by demyelination or through anterograde Wallerian degeneration. Additional histopathologic and advanced MR imaging studies evaluating larger series of patients will be necessary to better elucidate the role of CNS involvement in patients with pSS and to improve the understanding of the pathologic mechanisms behind the reduction of WM tract integrity in pSS.

\section{REFERENCES}

1. Fox RI, Howell FV, Bone RC, et al. Primary Sjogren syndrome: clinical and immunopathologic features. Semin Arthritis Rheum 1984;14:77-105

2. Mavragani CP, Moutsopoulos HM. The geoepidemiology of Sjogren's syndrome. Autoimmun Rev 2010;9:A305-10

3. Kassan SS, Moutsopoulos HM. Clinical manifestations and early diagnosis of Sjogren syndrome. Arch Intern Med 2004;164:1275-84

4. Soliotis FC, Mavragani CP, Moutsopoulos HM. Central nervous system involvement in Sjogren's syndrome. Ann Rheum Dis 2004;63:616-20

5. Fauchais AL, Magy L, Vidal E. Central and peripheral neurological 
complications of primary Sjogren's syndrome. Presse Med 2012;41:e485-93

6. Tobon GJ, Pers JO, Devauchelle-Pensec V, et al. Neurological disorders in primary Sjogren's syndrome. Autoimmune Dis 2012:645967

7. Drosos AA, Andonopoulos AP, Lagos G, et al. Neuropsychiatric abnormalities in primary Sjogren's syndrome. Clin Exp Rheumatol 1989;7:207-09

8. Tzarouchi LC, Tsifetaki N, Konitsiotis S, et al. CNS involvement in primary Sjogren syndrome: assessment of gray and white matter changes with MRI and voxel-based morphometry. AJR Am J Roentgenol 2011;197:1207-12

9. Alexander EL, Beall SS, Gordon B, et al. Magnetic resonance imaging of cerebral lesions in patients with the Sjogren syndrome. Ann Intern Med 1988;108:815-23

10. Morgen K, McFarland HF, Pillemer SR. Central nervous system disease in primary Sjogren's syndrome: the role of magnetic resonance imaging. Semin Arthritis Rheum 2004;34:623-30

11. Harboe E, Beyer MK, Greve OJ, et al. Cerebral white matter hyperintensities are not increased in patients with primary Sjogren's syndrome. Eur J Neurol 2009;16:576-81

12. Kao CH, Ho YJ, Lan JL, et al. Regional cerebral blood flow and glucose metabolism in Sjogren's syndrome. J Nucl Med 1998; 39:1354-56

13. Huang WS, Chiu PY, Kao A, et al. Detecting abnormal regional cerebral blood flow in patients with primary Sjogren's syndrome by technetium-99m ethyl cysteinate dimer single photon emission computed tomography of the brain: a preliminary report. Rheumatol Int 2003;23:174-77

14. Basser PJ, Pierpaoli C. Microstructural and physiological features of tissues elucidated by quantitative-diffusion-tensor MRI. J Magn Reson B 1996;111:209-19

15. Pierpaoli C, Jezzard P, Basser PJ, et al. Diffusion tensor MR imaging of the human brain. Radiology 1996;201:637-48

16. Segal BM, Mueller BA, Zhu X, et al. Disruption of brain white matter microstructure in primary Sjogren's syndrome: evidence from diffusion tensor imaging. Rheumatology 2010;49:1530-39

17. Astrakas LG, Argyropoulou MI. Shifting from region of interest (ROI) to voxel-based analysis in human brain mapping. Pediatr Radiol 2010;40:1857-67

18. Smith SM, Jenkinson M, Johansen-Berg H, et al. Tract-based spatial statistics: voxelwise analysis of multi-subject diffusion data. NeuroImage 2006;31:1487-505

19. Smith SM, Jenkinson M, Woolrich MW, et al. Advances in functional and structural MR image analysis and implementation as FSL. NeuroImage 2004;23(Suppl 1):S208-19

20. Nichols TE, Holmes AP. Nonparametric permutation tests for functional neuroimaging: a primer with examples. Hum Brain Mapping 2002;15:1-25

21. Smith SM, Nichols TE. Threshold-free cluster enhancement: addressing problems of smoothing, threshold dependence and localisation in cluster inference. NeuroImage 2009;44:83-98

22. Pierpaoli C, Barnett A, Pajevic S, et al. Water diffusion changes in Wallerian degeneration and their dependence on white matter architecture. NeuroImage 2001;13:1174-85

23. Kanaan RA, Shergill SS, Barker GJ, et al. Tract-specific anisotropy measurements in diffusion tensor imaging. Psychiatr Res 2006; 146:73-82
24. Mukherjee P. Diffusion tensor imaging and fiber tractography in acute stroke. Neuroimaging Clin North Am 2005;15:655-65

25. Basser PJ, Pajevic S, Pierpaoli C, et al. In vivo fiber tractography using DT-MRI data. Magn Reson Med 2000;44:625-32

26. Le Bihan D. Looking into the functional architecture of the brain with diffusion MRI. Nat Rev Neurosci 2003;4:469-80

27. Wheeler-Kingshott CA, Cercignani M. About "axial" and "radial" diffusivities. Magn Reson Med 2009;61:1255-60

28. Song SK, Sun SW, Ramsbottom MJ, et al. Dysmyelination revealed through MRI as increased radial (but unchanged axial) diffusion of water. NeuroImage 2002;17:1429-36

29. Song SK, Sun SW, Ju WK, et al. Diffusion tensor imaging detects and differentiates axon and myelin degeneration in mouse optic nerve after retinal ischemia. NeuroImage 2003;20:1714-22

30. Alexander EL. Neurologic disease in Sjogren's syndrome: mononuclear inflammatory vasculopathy affecting central/peripheral nervous system and muscle: a clinical review and update of immunopathogenesis. Rheum Dis Clin North Am 1993;19:869-908

31. Belin C, Moroni C, Caillat-Vigneron N, et al. Central nervous system involvement in Sjogren's syndrome: evidence from neuropsychological testing and HMPAO-SPECT. Ann Med Interne 1999;150: 598-604

32. Johnson AC, McNabb NA, Rossiter RJ. Chemistry of Wallerian degeneration: a review of recent studies. Arch Neurol Psychiatry 1950;64:105-21

33. Lampert PW, Cressman MR. Fine-structural changes of myelin sheaths after axonal degeneration in the spinal cord of rats. $A m ~ J$ Pathol 1966;49:1139-55

34. Thomalla G, Glauche V, Koch MA, et al. Diffusion tensor imaging detects early Wallerian degeneration of the pyramidal tract after ischemic stroke. NeuroImage 2004;22:1767-74

35. Misu T, Fujihara K, Kakita A, et al. Loss of aquaporin 4 in lesions of neuromyelitis optica: distinction from multiple sclerosis. Brain 2007;130:1224-34

36. Estiasari R, Matsushita T, Masaki K, et al. Comparison of clinical, immunological and neuroimaging features between anti-aquaporin-4 antibody-positive and antibody-negative Sjogren's syndrome patients with central nervous system manifestations. Mult Scler 2012;18:807-16

37. Sofat N, Venables PJ. Is Sjogren myelopathy Devic disease? Ann Rheum Dis 2008;67:730-31

38. Nitescu D, Nicolau A, Caraiola S, et al. Neuromyelitis optica: complication or comorbidity in primary Sjogren's syndrome? Rom J Intern Med 2011;49:295-300

39. Bjartmar C, Yin X, Trapp BD. Axonal pathology in myelin disorders. J Neurocytol 1999;28:383-95

40. Ichikawa H, Ishihara K, Fujimoto R, et al. An autopsied case of Sjogren's syndrome with massive necrotic and demyelinating lesions of the cerebellar white matter. J Neurol Sci 2004;225:143-48

41. Yaguchi H, Houzen H, Kikuchi K, et al. An autopsy case of Sjogren's syndrome with acute encephalomyelopathy. Intern Med 2008;47: 1675-80

42. Rueda Lopes FC, Doring T, Martins C, et al. The role of demyelination in neuromyelitis optica damage: diffusion-tensor MR imaging study. Radiology 2012;263:235-42

43. Yu C, Lin F, Li K, et al. Pathogenesis of normal-appearing white matter damage in neuromyelitis optica: diffusion-tensor MR imaging. Radiology 2008;246:222-28 